\title{
Seeking the solution
}

\author{
Is there any fundamental \\ reason to be fixated on water \\ as the universal elixir of life? \\ Philip Ball investigates.
}

$\mathbf{W}$ here there's water, there's life. That, at least, is what our experience on Earth has taught us, and when it comes to searching for life on other worlds, NASA seems determined to follow the water. But is it right to see water as the sole medium for extraterrestrial life?

Some think not. "Water is a terrible solvent for life," says chemist Steven Benner of the University of Florida in Gainesville. Benner is one of a number of biochemists, planetary scientists and philosophers who are trying to find out whether water is in some sense 'finetuned' for life.

It is a pertinent question. Scientists today are searching for extraterrestrial life wherever they can, from the subsoil of Mars to planets orbiting other stars. So far they have found nothing. But are they looking in the right places? If Benner is right, perhaps they have become too fixated on chasing water.

Benner led the case for the prosecution earlier this year at a meeting in Varenna, Italy. There, researchers faced up to what many of them consider to be their biggest challenge. How can we even begin to discuss the relationship between water and life when we have only one example - life on Earth. And more to the point, can the problem be dealt with in a rigorous, scientific manner?

For Benner the answer to the latter question is yes. He argues that it is possible to investigate experimentally whether water is essential for life. He hopes to prove that a type of biochemistry can occur without water. "We are working to create alternative darwinian systems based on fundamentally different chemistries," he says. "We are using different solvent systems as a way to get a precursor for life on Earth."

The notion of redesigning life's chemistry has become central to the emerging discipline of synthetic biology, which has among its longterm objectives the aim of creating entire cells from scratch, perhaps with a different chemical basis from that of existing organisms.

\section{IMAGE \\ UNAVAILABLE FOR COPYRIGHT REASONS}

Benner points out that water is generally not a good solvent for doing organic chemistry which is, in the end, what life is all about. For one thing, water is rather reactive, tending to split apart the bonds that link the building blocks of biomolecules together. It readily breaks peptide bonds, for example, as well as many of the bonds in nucleic acids, such as RNA. "The structure of RNA screams 'I did not arise in water!'” Benner asserts. He says that in about four out of five cases, synthetic organic chemists will avoid using water as a solvent.

\section{Creative force}

But of course organic chemists aren't usually trying to create life. Water has many properties that seem indispensable for the functioning of proteins and cells. It is an excellent solvent for ions, for example - crucial for nerve signalling, enzymatic processes, biomineralization and the behaviour of DNA. It is also a master of weak intermolecular interactions such as hydrogen bonds and hydrophobic forces. The latter play a central role in protein folding and protein-protein interactions, whereas the former often act as bridges between protein binding sites and their substrates. And water's ability to absorb and lose heat without undergoing a large temperature change provides thermal cushioning, shielding cells and organisms from wild temperature swings.

No other known liquid combines all of these properties. But does a life-supporting solvent need them all? Are any of water's unique prop- erties essential, and are any of its essential $\overrightarrow{\tilde{v}}$ properties unique?

To assess whether water is somehow 'special' as a biological solvent, we need to understand the basic requirements for life ${ }^{1}$. Proteins and nucleic acids rely on weak intermolecular interactions to organize and pass chemical information around - to transfer genetic instructions, for instance. It could be argued that general properties such as this will be needed for a 'chemistry of life', even when the building blocks are not proteins or nucleic acids.

But these familiar building blocks may themselves not need water to function. "I think it is perfectly possible that at least elements of relevant biochemistry can be persuaded to work in a completely non-aqueous environment," says physicist John Finney at University College London.

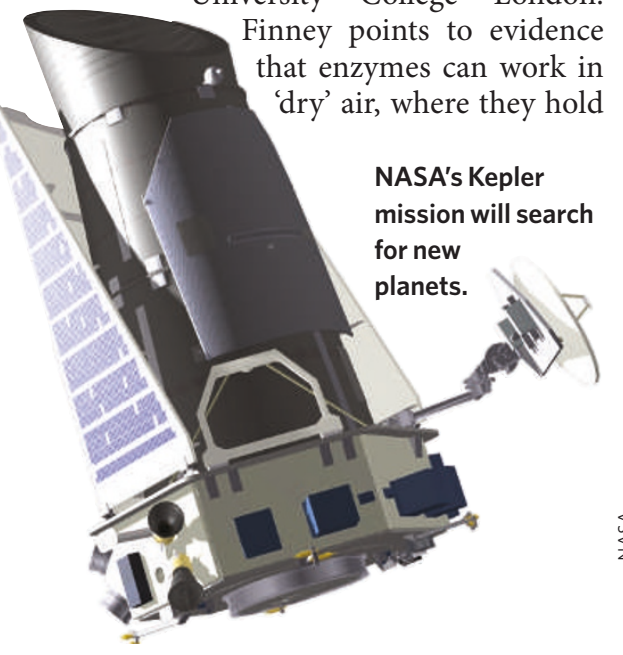


IMAGE

\section{UNAVAILABLE FOR COPYRIGHT} REASONS

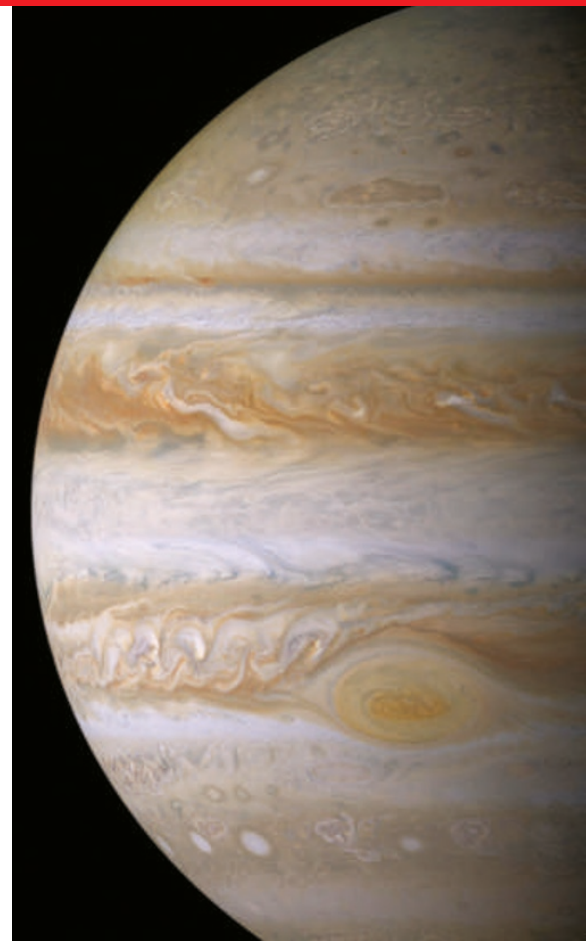

The clouds of Jupiter contain liquid ammonia which some say could support water-free life.

cosmologists, the physical Universe seems to be precariously fine-tuned to make life possi$\mathrm{ble}^{5}$. For example, the fine-structure constant, which determines the strength of electromagnetic interactions, is not fixed by any known fundamental theory; and yet if it was ten times larger, stable atoms could not exist.

Unlike physics, of course, biochemistry adapts to its environment, which is why the participants in Varenna generally agreed that life on Earth is adapted to water rather than the other way round. "Life on Earth itself is fine-tuned to water - a consequence of it evolving in close association with the medium," says Finney. "To put it the other way is perhaps to put the cart before the horse." He adds that "the fine-tuning argument with respect to water is a far more
"Life on Earth is fine-tuned to water - a consequence of it evolving in close association with the medium." - John Finney formamide, which is liquid over a wide range of temperatures and pressures, dissolves salts and has hydrophobic-like effects. Formamide might be present below the surface on Mars.

But Benner does not rule out more exotic possibilities, such as liquid nitrogen or supercritical liquid hydrogen on gas-giant planets such as Saturn, Uranus and Neptune. Or perhaps hydrocarbons such as liquid methane on Titan. "Organic reactivity in hydrocarbon solvents is no less versatile than in water," he says.

Does widening the search for extraterrestrial life to places that don't have water make sense? The Cassini-Huygens space probe showed in January that Titan may have riverbeds (if not actual rivers) carved from liquid hydrocarbons. And in March the detection of reflected light from two Jupiter-like extrasolar planets by the Spitzer Space Telescope $e^{7}$ marked the first step towards analysing their chemistry remotely.

But NASA's quest for habitable planets remains focused on water. In 2008 it intends to launch the Kepler Photometer, which will search for Earth-like planets by looking for evidence of their transit across the faces of parent stars. At a much later date, the agency hopes to launch two space-based telescopes that will form the Terrestrial Planet Finder (TPF) mission. These will detect and analyse reflected light from other planets. The gaze of both Kepler and the TPF will be firmly fixed on the 'habitable zone' of stars, where liquid water could exist, potentially overlooking worlds that are habitable by non-aqueous life forms.

Benner is not waiting around for these space missions to find extraterrestrial life in places with or without water. $\mathrm{He}$ is convinced that the time is ripe to explore more exotic life forms in the laboratory. But that, he says, requires a different mindset from the one that currently guides chemical research and funding. Benner is participating in a US National Academies panel funded by NASA that is looking at possible alternative complex problem than that in astrophysics. Without knowing what aspects of water are important, I suspect we are doing little more than speculating."

\section{Out of this world}

Benner wants to use experiments to move beyond these abstract discussions. He sees several alternatives to water ${ }^{6}$. Ammonia, which is liquid between $-78^{\circ} \mathrm{C}$ and $-33^{\circ} \mathrm{C}$ at 1 atmosphere pressure, will dissolve many organic compounds and can form hydrogen bonds. It is also relatively common in the cosmos: there is liquid ammonia in the clouds of Jupiter, for example. Benner considers water-ammonia mixtures, which may exist in some cold extraterrestrial environments such as beneath the surface of Saturn's moon Titan, as another promising candidate. Then there is chemistries for life, and which he hopes will identify research directions that funding agencies can pursue. He believes that researchers should aim high - to create life forms that do not reproduce the chemistry that is found on Earth. In other words, if we can't easily get to other worlds, we should build them here. Philip Ball is a consultant editor for Nature.

Benner S. A. \& Ellington, A. D.CRC Crit Rev. Biochem. 23, 369-426 (1988)

2. Lee, M.-Y. \& Dordick, J. S. Curr. Opin. Biotechnol. 13, 376-384 (2002)

3. Bergman, D. L. \& Lynden-Bell, R. M. Mol. Phys. 99, 1011-1021 (2001).

4. Lynden-Bell, R. M. \& Debenedetti, P. G. J. Phys. Chem. B 109, 6527-6534 (2005)

5. Hogan, C. J. Rev. Mod. Phys. 72, 1149-1161 (2000)

6. Benner, S. A., Ricardo, A. \& Carrigan, M. A. Curr. Opin Chem. Biol. 8, 672-689 (2004).

7. Deming, D., Seager, S., Richardson, L. J. \& Harrington, J. Nature 434, 740-743 (2005) strange to biologists and chemists, but it is far more common in cosmology or physics. For

\section{(a)}

